\title{
Double blind, you have been voted off the island!
}

"Double blind" is the term researchers frequently use, and readers frequently accept, as a key marker of the validity of a randomised controlled trial (RCT). Clinical trial experts and clinicians, when asked, are all ready to offer a definition of what double blind means. Unfortunately, however, their answers differ substantially. ${ }^{1}$ Thus, the term lacks consistency in its use and interpretation, a critical flaw for any technical term. In this editorial, we advocate abandoning the current blinding lexicon (ie, single, double, and triple blinding) and recommend transparent reporting of the blinding status of each group involved in the execution, monitoring, and reporting of clinical trials.

Blinding (or masking) in RCTs is the process of withholding information about treatment allocation from those who could potentially be influenced by this information. Blinding has long been considered an important safeguard against bias. Benjamin Franklin, in 1784, was probably the first to use blinding in scientific experimentation. ${ }^{2}$ Louis XVI commissioned Franklin to evaluate mesmerism, the most popular unconventional "healing fluid" of the eighteenth century. ${ }^{2}$ By applying a blindfold to participants Franklin removed their knowledge of when mesmerism was and was not being applied. Blinding eliminated the intervention's effects and established mesmerism as a sham. ${ }^{2}$ From this work, the scientific community recognised the power of blinding to enhance objectivity, and it quickly became, and remains, a commonly used strategy in scientific research.

The groups who can potentially introduce bias into an RCT through knowledge of the treatment allocation are shown in the table.

Individuals in the 7 groups in the table are likely to have, or to develop, opinions about the efficacy of the intervention being investigated. Because of these opinions, unblinded individuals can systematically bias trial findings through conscious or unconscious mechanisms. When unblinded, participants may introduce bias through use of other effective interventions, differential reporting of symptoms, ${ }^{3}$ psychological or biological effects of receiving a placebo (although recent studies show conflicting evidence), ${ }^{45}$ or dropping out. Unblinded healthcare providers can distort trial results if they differentially prescribe effective co-interventions, influence compliance with follow up, or influence patient reports. ${ }^{3}$ Unblinded data collectors can introduce bias through differential encouragement during performance testing, differential timing or frequency of outcome measurements, and variable recording of outcomes. ${ }^{6}$ Unblinded judicial assessors may introduce bias in their assessments of outcomes, this being most likely during assessment of subjective outcomes. ${ }^{3}$ Unblinded data analysts have the potential to introduce systematic bias through decisions on patient withdrawals, post hoc selection of outcomes or analytic approaches, selection of time points that show the maximum or minimum effects, and many other decisions. ${ }^{8}$ Unblinded members of the data safety and monitoring committee may introduce bias at the time of interim analyses through their recommendations to stop or continue a study. ${ }^{8}$ Blinding of authors, while seldom done, ${ }^{89}$ may reduce the bias in the presentation and interpretation of results.

Case reports document individual examples of the biases described above. ${ }^{781011}$ However, no high quality methodological studies have been done to evaluate whether blinding of individual groups systematically affects the estimate of effect in RCTs. Investigators have published 2 high quality methodological studies (ie, studies that assessed RCTs from meta-analyses, thereby controlling for the confounders of disease state and intervention), but they assessed the influence of investigators' statements that the trials were double blinded on the estimate of effect. ${ }^{12}{ }^{13}$ Although one study showed lower estimates of effect in RCTs reported as double blinded, ${ }^{12}$ the other study did not find any association between the reporting of double blinding and the magnitude of effect. ${ }^{13}$ Who was actually blinded in these studies probably varied and is certainly open to question. Heterogeneity in who was blinded in the studies reported as double blinded may be responsible for these discrepant findings.

Although the true magnitude of bias introduced by unblinding remains (and is likely to remain) uncertain, clinicians should consider the blinding status of each group in assessing study validity. Unfortunately, suboptimal reporting of blinding status in full text publications and secondary journals has hindered readers. ${ }^{14}{ }^{15}$ Authors have commonly relied on conventional blinding terminology (single, double, and triple blinding) to convey blinding status. ${ }^{1}$ We have shown great variability in physician interpretations and textbook definitions of these terms. ${ }^{1}$ It is for this reason that we recommend, and the editors of $A C P$ Journal Club, Evidence-Based Medicine, Evidence-Based Mental Health, and Evidence-Based Nursing have adopted, a strategy of

Groups that can potentially be blinded in RCTs

\begin{tabular}{ll}
\hline Participants & Individuals who are randomly assigned to the interventions being evaluated \\
\hline Healthcare providers & $\begin{array}{l}\text { The physicians, nurses, physiotherapists, or other personnel who care for the participants during the study } \\
\text { period or administer the interventions }\end{array}$ \\
\hline Data collectors & $\begin{array}{l}\text { Individuals who collect data for the study outcomes. Data collection could include administering a questionnaire, } \\
\text { taking a physical measurement, or eliciting symptoms }\end{array}$ \\
\hline Judicial assessors of outcomes & The individuals who ultimately decide whether a participant has had the outcome, or outcomes, of interest \\
\hline Data analysts & The individuals who conduct the data analysis \\
\hline Manuscript writers & $\begin{array}{l}\text { The committee that reviews the data to advise on the continuing safety of the trial and persistent uncertainty of } \\
\text { the efficacy of the intervention being evaluated }\end{array}$ \\
\hline & $\begin{array}{l}\text { The individuals who write versions of the manuscript before the breaking of the randomisation code: in a fully } \\
\text { blinded study, one version is written with the assumption that group A received the experimental intervention, } \\
\text { and the other is written with the assumption that group B received the experimental intervention }\end{array}$ \\
\hline
\end{tabular}


abandoning the current blinding terminology for transparent reporting of the blinding status of the groups listed in the table. As a result of this policy, readers will be able to make more informed decisions about the validity of the studies upon which they base their practice.

Although our editorial focuses on blinding in RCTs, the issue is also relevant for other study designs where knowledge of group membership may affect the results. For example, in cohort or case control studies where charts are abstracted by researchers to find outcomes or predisposing factors, readers should look to determine if the abstractors were blinded to group membership. Unblinded data abstractors may lead to variable recording of outcomes or predisposing factors. As in RCTs, blinding is not always possible. Notwithstanding the feasibility of blinding, readers should, when assessing the validity of a study, consider the blinding status of all groups who may introduce bias through knowledge of group membership.

P J DEVEREAUX, MD

MOHIT BHANDARI, MD, MSC

McMaster University

Hamiltom, Ontario, Canada

VICTOR M MONTORI, MD, MSC

Mayo Clinic

Rochester, Minnesota, USA

BRADEN J MANNS, MD

WILLIAM A GHALI, MD, MPH

University of Calgary

Calgary, Alberta, Canada

GORDON H GUYATT, MD, MSC

McMaster University

Hamilton, Ontario, Canada
1 Devereaux PJ, Manns BJ, Ghali WA, et al. Physician interpretations and textbook definitions of blinding terminology in randomized controlled trials. JAMA 2001;285:2000-3.

2 Kaptchuk TJ. Intentional ignorance: a history of blind assessment and placebo controls in medicine. Bull Hist Med 1998;72:389-433.

3 Altman DG, Schulz KF, Moher D, et al. The revised CONSORT statement for reporting randomized trials: explanation and elaboration. Ann Intern Med 2001;134:663-94.

4 de la Fuente-Fernandez R, Ruth TJ, Sossi V, et al. Expectation and dopamine release: mechanism of the placebo effect in Parkinson's disease. Science 2001;293:1164-6.

5 Hróbjartsson A, Gøtzsche PC. Is the placebo powerless? An analysis of clinical trials comparing placebo with no treatment. $N$ Engl I Med 2001;344:1594-1602. Review: http://www.ebmentalhealth.com/cgi/ content/full/5/1/15

6 Jadad AR. Randomised controlled trials: a user's guide. London, England: BMJ Books;1998:20-36.

7 Guyatt GH, Pugsley SO, Sullivan MJ, et al. Effect of encouragement on walking test performance. Thorax 1984;39:818-22.

8 Gøtzsche PC. Blinding during data analysis and writing of manuscripts. Control Clin Trials 1996;17:285-93.

9 Dong BJ, Hauck WW, Gambertoglio JG, et al. Bioequivalence of generic and brand-name levothyroxine products in the treatment of hypothyroidism. JAMA 1997;277:1205-13.

10 Karlowski TR, Chalmers TC, Frenkel LD, et al. Ascorbic acid for the common cold. A prophylactic therapeutic trial. JAMA 1975;231:1038-42.

11 Noseworthy JH, Ebers GC, Vandervoort MK, et al. The impact of blinding on the results of a randomized, placebo controlled multiple sclerosis clinical trial. Neurology 1994;44:16-20.

12 Schulz KF, Chalmers I, Hayes RJ, et al. Empirical evidence of bias. Dimensions of methodological quality associated with estimates of treatment effects in controlled trials. JAMA 1995;273:408-12.

13 Moher D, Pham B, Jones A, et al. Does quality of reports of randomised trials affect estimates of intervention efficacy reported in meta-analyses? Lancet 1998;352:609-13.

14 Devereaux PJ, Manns BJ, Ghali WA, et al. The CONSORT checklist as a means to enhance completeness of reporting in randomized controlled trials [Abstract]. Clin Invest Med 2000;23(Suppl):S12.

15 Devereaux PJ, Manns BJ, Ghali WA, et al. Reviewing the reviewers: the quality of reporting in three secondary journals. CMAJ 2001;164:1573-6. 